\title{
Content Based Image Retrieval for Unlabelled Images
}

\author{
Rikin K Thakkar, Ms Ompriya Kale, \\ Dept of computer engineering Lok Jagruti Kendra Institute of Technology, India. \\ Ass Prof Dept of Information Technology, Lok Jagruti Kendra Institute of Technology, India.
}

\begin{abstract}
Recently, content-based image retrieval has become hot topic and the techniques of content-based image retrieval have been achieved good development. Content-based image retrieval systems were introduced to address the problems associated with text-based image retrieval. In this paper, basic components of contentbased image retrieval system are introduced here. Images are classified as lablled and unlablled images. Here survey on content based image retrieval given with some Image retrieval methods based on unlabelled data like D-EM, SVM, Relevance Feedback, Semi-Supervised/Active Learning, Transductive Learning, Bootstrapping SVM, Active learning, SSMIL and Label propagation Methods are presented in this paper. Comparison of these all methods is also presented in this paper.
\end{abstract}

Keywords: Image retrieval, CBIR, Unlabelled images, SVM

\section{Introduction}

IBM was the first, who take an initiative by pro-posing query-by image content (QBIC). QBIC developed at the IBM Almaden Research Center is an open frame-work and development technology. Fig.2 shows general Framework of Content based Image Retrieval system. All images will undergo the low level feature extrac-tion process before being added to the images database. In feature extraction stage, features such as colour, shape or texture are extracted from the image. User provides a sample image and the similarity measurement engine is responsible in estimating the similarity between the query image and database images and then ranking them ac-cording to their similarity to the given query image. ${ }^{[34]}$

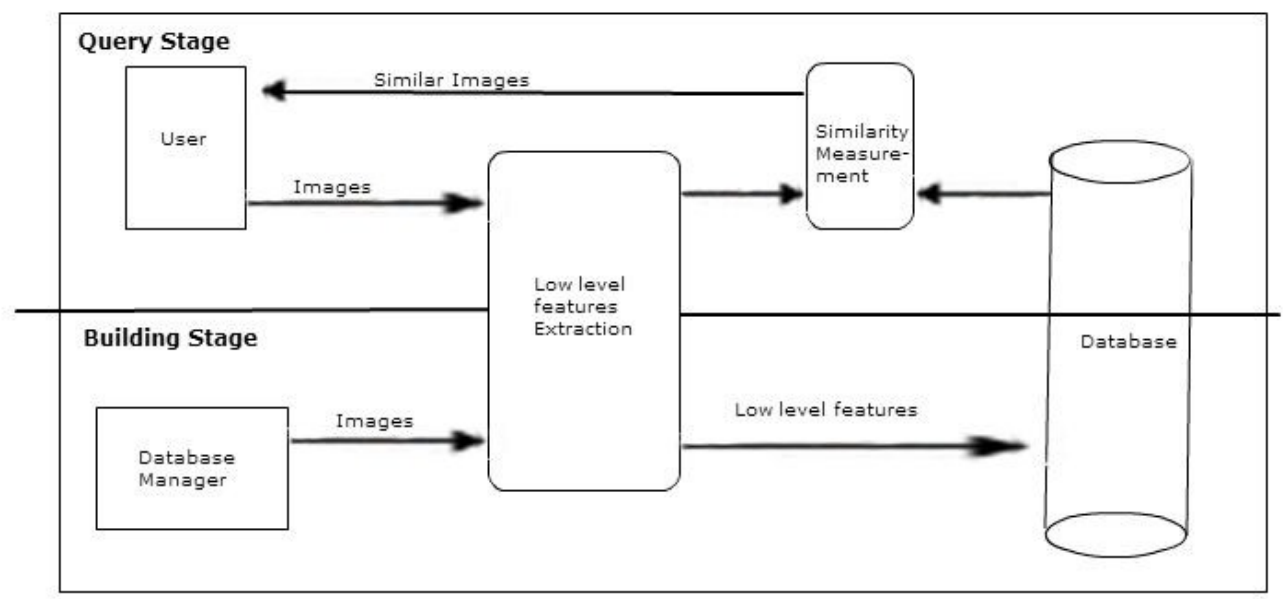

Fig 1. General Framework of Content Based Image Retrieval ${ }^{[34]}$

Images are classified as labeled and unlabelled images. But this classification is confronted by two main challenges. The first one is that the labelled training samples are too limited. We cannot give label to each and every image. Generally, the labels are provided by user's queries and relevance feedbacks, which will be very limited. Limited training data would only result in weak classification ${ }^{[33]}$. Another challenge is the dimensionality of learning, since high dimensional visual data would pose practical difficulties for feature weighting, selecting and dimensionality reduction. Limited training data would also prevent effective dimensionality reduction schemes. If there are a large number of unlabelled images in the given database, we may use them to boost the weak classifier learned from the limited labelled data, since unlabelled data contain information about the joint distribution over features ${ }^{[2,3,33]}$. The whole image is not interesting to the user, only a very small portion of the large image database is interesting to the user, in which almost images are unlabelled. Much work regards the problem as a strict two class classification problem, with equal treatments on both 
positive and negative examples. Positive examples cluster in certain way, but negative examples usually do not cluster since they can belong to any class. It is almost impossible to estimate the real distribution of negative images in the database based on the relevant feedback ${ }^{[4,5,33]}$. A significant stage in the development of CBIR system is low level feature extraction. Feature extraction may be done from region or an entire image ${ }^{[9,10]}$. The Bayes point machines approach used to give the soft annotation to the unlabelled image ${ }^{[11]}$. The section-II of this paper presents related work of image classification with unlabelled image. Section-III of this paper gives conclusion.

\section{Related Work}

Here some methods of content based image retrieval based on unlabelled data is explained in brief. Also the comparison of each method is given at the end of this section.

\section{A. D-EM (Discriminant-EM) Algorithm}

The D-EM algorithm ${ }^{[14,15]}$ is used as a way of exploiting an unlabelled data in Content Based Image Retrieval system. This is three steps iterative method for an efficient way of retrieving an unlabelled image. The three steps are E-step, D-step, and M-step respectively. In such circumstance, the hybrid training data set D consists of a labelled data set $\mathrm{L}=\{(\mathbf{x i}, \mathrm{yi}), \mathrm{i}=1, ., \mathrm{N}\}$, and an unlabelled data set $\mathrm{U}=\{\mathbf{x i}, \mathrm{i}=1, ., \mathrm{M}\}$. In CBIR, the query images act as the labelled data, and the whole database or a subset can be treated as the unlabelled set ${ }^{[16]}$. We make an assumption here that $\mathrm{L}$ and $\mathrm{U}$ are from the same distribution. Therefore, image retrieval is to classify the images in the database based on both labelled and unlabelled training data. The applied to this learning task, since the labels of unlabelled data can be treated as missing values ${ }^{[17]}$. Finally D-EM algorithm ${ }^{[18]}$ is working an accuracy without than unlabelled data in image database. This algorithm is give guideline for the system to utilize the unlabelled data with labelled data. ${ }^{[33]}$

\section{B. Support Vector Machines}

Support Vector Machines have shown their capacities in pattern recognition. The aim of SVM classification method is to find the best hyper-plane separating relevant and irrelevant vectors maximizing the size of the margin (between both classes). Initial method assumes that relevant and irrelevant vectors are linearly separable ${ }^{[19]}$. The SVM separate the whole image database into two classes. The two classes are also including the unlabelled images with two types they are relevant and irrelevant unlabelled images. The relevant unlabelled image is related to the relevant labelled images in the image database. In similar way the irrelevant unlabelled image is related to the irrelevant labelled images in the database. This SVM is also classifying the unlabelled images in accuracy manner. ${ }^{[33]}$

\section{Relevance Feedback}

As image databases usually contain unlabelled images, these can be exploited to help supervised learning by asking the user to label them. The goal is naturally to minimize asking help from the user. The relationships of all the data points in the feature space using a manifold ranking algorithm and constructs a weighted graph that contains all images; the ranking scores of labelled examples are iteratively propagated to nearby labelled and unlabelled images ${ }^{[20]}$. The Relevance Feedback is using the likelihood functions used to relate the certain data points in the image class. These function is define the relevancy of each data point to the user given query. This is most useful to give rank to unlabelled images in the image database ${ }^{[21,22]}$. The learners are trained with labelled and unlabelled images. Then the process is merged in form of positive image. That image having the high confidence degree for presenting the required user in the result form. ${ }^{[33]}$

\section{Semi-Supervised/Active Learning}

This learning ${ }^{[23]}$ is working with two different learners for training the retrieval process of unlabelled image by using labelled images from image database. The learning is dividing into two types, semi-supervised and active. Both are different. Here most important result of learners opinion of unlabelled images. And also using some sampling process, these samples are mainly used to select the unlabelled images during the image retrieval. Semi-supervised learning ${ }^{[24]}$ deals with methods for exploiting unlabelled data in addition to labelled data to improve learning performance. Two independent sets of attributes, and uses the prediction of each learner on unlabelled examples to augment the training set of the other ${ }^{[25]}$. Active learning deals ${ }^{[26]}$ with methods that assume the learner has some control over the input space. In utilizing unlabelled data, it goes a different way from semisupervised learning, with the goal of minimizing the number of queries required. There are two major schemes, i.e. uncertainty sampling train a single learner and then query the unlabelled instances on which the learner is the least confident. Committee-based sampling generate a committee of several learners and select the unlabelled instances on which the committee members disagree the most ${ }^{[27,33]}$. 


\section{E. Transductive Learning Method}

In this learning method ${ }^{[29]}$ is processing the unlabelled image with the probability manner. The high probability is used to identify the unlabelled images from the whole image database. The Ranking Process The resultant ranking score of an unlabelled image is in proportion to the probability that it is relevant to the query, with large ranking score indicating high probability. Thus the effect of unlabelled data is gradually incorporated into the ranking score ${ }^{[30,33]}$.

\section{F. Bootstrapping SVM active learning}

The normal SVM active learning is used. When active learning ends, the database images are ranked in descending order of the distances to the final separating hyperplane, and the top images are shown as the retrieval result. It aims to achieve good classification performance with the help of unlabelled data in the presence of the small sample problem. The labelled images consist of at least one positive and one negative image. Then the unlabelled images in the image database are incorporated to train the initial SVM classifier. The initial classifier is improved by incorporating unlabelled images instead of heuristically labelling more images. Hence, it reduces the labelling cost and thus the burden on the user to improve the retrieval. This method is general such that any algorithm for learning from labelled and unlabelled data can be incorporated ${ }^{[2,33,31]}$

\section{G. Semi-Supervised Multiple Instance Learning (SSMIL)}

The Formulation straightforward way is to map both the labelled and unlabelled bags into the feature space determined by all the labelled bags. The Up-Speed after each labelled bag is mapped into the feature space; all the unlabelled bags can also be mapped into this feature space. Hence, the dimension for each bag can be greatly reduced, with the irrelevant features being discarded. To select the most important instances and mapping each bag $\mathbf{B}$ in both the labelled and unlabelled set into the space determined by these instances as follows ${ }^{[33,27]}$.

TABLE-I SUMMARY OF PREVIOUS PROCESS ${ }^{[33]}$

\begin{tabular}{|l|l|l|}
\hline $\begin{array}{l}\text { SR } \\
\text { NO. }\end{array}$ & Process Name & Description \\
\hline 1 & D-EM(Discriminant-EM)Algorithm & Unlabelled image as data set \\
\hline 2 & Support Vector Machines & $\begin{array}{l}\text { Unlabelled image as two classes } \\
\text { relevant and irrelevant }\end{array}$ \\
\hline 3 & Relevance Feedback & Unlabelled image having rank \\
\hline 4 & Semi-Supervised/Active Learning & Unlabelled image as training set \\
\hline 5 & TRANSDUCTIVE LEARNING METHOD & Unlabelled image as ranking score \\
\hline 6 & Bootstrapping SVM active learning & $\begin{array}{l}\text { Unlabelled image as initial SVM } \\
\text { classifier }\end{array}$ \\
\hline 7 & Semi-Supervised Multiple Instance Learning (SSMIL) & $\begin{array}{l}\text { Unlabelled image as instance of } \\
\text { mapped bag }\end{array}$ \\
\hline
\end{tabular}

\section{Conclusion}

In this paper we surveyed the content-based image retrieval system, by providing an overview of the most important aspects characterizing that kind of images. The image databases are classified into the labelled image and unlabelled image. In this paper, we have presented a survey on content based image retrieval based on unlabelled images. We compare the many image database classification processes based on unlabelled images. The image classifying processes are more useful to the next generation of the content based image retrieval system with unlabelled images.

\section{References}

[1]. Jain and A.Vailaya, "Image Retrieval Using Color and Shape,”Pattern Recognit., Vol. 29, No. 8, pp. 1233-1244, Aug.1996.

[2]. Lei Wang, KapLuk Chan, Zhihua Zhang, "Bootstrapping SVM Active Learning by Incorporating Unlabelled Images for Image Retrieval." Lining Zhang, Student Member, IEEE, Lipo Wang, Senior Member, IEEE, and Weisi Lin*, Senior Member, IEEE. "Conjunctive Patches Subspace Learning with Side Information for Collaborative Image Retrieval."

K. Tieu and P. Viola, "Boosting image retrieval," Int. J. Comput. Vis.,vol. 56, no. 1/2, pp. 17-36, 2004.

[4]. R. Anderson, “A spreading activation theory of memory," VerbalLearning and Verbal Behavior, 1983, 22: $261-295$.

[5]. I.J. Cox, et al., "The Bayesian image retrieval system, PicHunter: theory, implementation, and psychophysical experiments." IEEE Trans. On Image Processing. 9. (1)2000 p. 20-37.

[6]. JormaLaaksonen, Markus Koskela, Sami Laakso, ErkkiOja. "PicSOM- Content Based Image Retrieval with Self Organizing Maps." Pattern Recognition Letters 21 (2000) 1199-1207.

[7]. Koikkalainen, P., Oja, E., 1990. "Self-organizing hierarchical feature maps.” In: Proceedings of 1990 International Joint Conference on Neural Networks, Vol. II. IEEE, INNS, San Diego, CA.

[8]. MehwishRehman, Muhammad Iqbal, Muhammad Sharif and MudassarRaza. "Content Based Image Retrieval: Survey.”DOI: 10.5829/idosi.wasj.2012.19.03.1506, World Applied Sciences Journal 19 (3): 404-412, 2012, ISSN 1818-4952; IDOSI Publications, 2012.

[9]. Manjunath, B.S., and Ma, W.Y. "Texture features for browsing and retrieval of image data."IEEE Trans. OnPattern Analysis and Machine Intelligence, vol. 18, pp. 837- 842, 1996. 
[10]. C. Nastar, M. Mitschke and C. Meilhac "Efficient Query Refinement for Image Retrieval", IEEE Conf. Computer Vision and Pattern Recognition CVPR'98, Santa Barbara, CA, June 1998.

[11]. Songhao Zhu, Baoyun Wang, Yuncai Liu. "Spreading Activation Theory based image annotation." 978-1-4673-0046-9/12/ IEEE 2012 .

[12]. Smith, J.R., Chang, S.-F., 1996. "VisualSEEk: a fully automated content-based image query system.” In: Proceedings of the ACM Multimedia 1996. Boston, MA.

[13]. X.S. Zhou and T.S. Huang. "Unifying keywords and contents for image retrieval," in proc. International Workshop on ContentBased Multimedia Indexing. Italy. Sept. 2001.

[14]. Y.Wu, T.S.Huang, "Using Unlabelled Data in Supervised Learning by Discriminant-EM Algorithm",NIPS99 Workshop on Using Unlabelled Data in Supervised Learning, Colorado, 1999.

[15]. K. Nigam, A McCallum, S. Thrun, and T. Mitchell, "Text classification from labelled and unlabelled documents using EM," Machine Learning, 39, pp.1-32, 2000.

[16]. Wu, Y., Tian, Q., Huang, T.S. "Discriminant-EM algorithm with application to image retrieval." In: Proceedings of the IEEE International Conference on Computer Vision and Pattern Recognition, Hilton Head, SC (2000) 222-227.

[17]. Wu, Y., Tian, Q., and Huang, T. "Discriminant-EM algorithm with application to image retrieval." Proc. IEEE Conf. onComputer Vision and Pattern Recognition, vol. 1, pp. 155-162,

[18]. 2000

[19]. Y. Wu, Q. Tian, T. S. Huang, "Discriminant EM Algorithm with Application to Image Retrieval", IEEE Conf.Computer Vision and Pattern Recognition (CVPR)'2000, Hilton Head Island, South Carolina, June 13-15, 2000.

[20]. Bart Thomee, Michael S. Lew, "Relevance Feedback in Content- Based Image Retrieval: Promising Directions".

[21]. Campbell. "Algorithmic approaches to training support vector machines: A survey." In ESANN, pages 27-36. D-Facto Publications, 2000 .

[22]. G. Das and S. Ray. "A comparison of relevance feed-back strategies in CBIR," IEEE, 2007.

[23]. X.S. Zhou and T.S. Huang. "Exploring the nature andvariants of relevance feedback," in proc. IEEE Workshop onContent-based Access of Image and Video Libraries(CBAIVL), in conjunction with CVPR. Hawaii. Dec. 2001.

[24]. Cohen, N. Sebe, F. G. Cozman, M. C. Cirelo, and T. S. Huang, "Semi-supervised learning of classifiers: theory and algorithms for Bayesian network classifiers and applications to human-computer interaction," submitted to IEEE Trans. on PAMI, in revision, 2003.

[25]. Dan Zhang, Zhenwei Shi, Yangqiu Song, and Changshui Zhang. "Localized Content-Based Image Retrieval UsingSemi-Supervised Multiple Instance Learning."Y. Yagi et al. (Eds.): ACCV 2007, Part I, LNCS 4843, pp. 180-188,@ Springer-Verlag Berlin Heidelberg (2007).

[26]. Goldman, S., Zhou, Y. "Enhancing supervised learning with unlabelled data.”In:Proceedings of the 17th International Conference on Machine Learning, San Francisco, CA (2000) 327-334.

[27]. Tao Qin, Xu-Dong Zhang, Xu-Dong Zhang, De-Sheng Wang, Wei-Ying Ma, Hong-Jiang Zhang. “An active feedback framework for image retrieval."Elsevier 2007.

[28]. Ulges, M. Worring, and T. Breuel. "Learning Visual Contexts for Image Annotation from Flickr Groups." IEEE Transactions on Multimedia, 2011, 13(2): 330-341.

[29]. Jingrui He, Mingjing Li, Hong-Jiang Zhang, Hanghang Tong, Changshui Zhang. "Manifold-Ranking Based Image Retrieval."MM'04, NEW YORK, NEW YORK, USA. Copyright 2004 ACM 1-58113- 893-8/04/0010(OCTOBER 10-16, 2004).

[30]. Thomas S. Huang, Xiang Sean Zhou, MunehiroNakazato, Ying Wu, Ira Cohen. "Learning in Content-Based Image Retrieval."

[31]. Yunqiang Chen, Xiang Zhou, and Thomas S. Huang. "ONECLASS SVM FOR LEARNING IN IMAGE RETRIEVAL." In Proc. IEEE Int'l Conf. on Image Processing, Thessaloniki, Greece (2001).

[32]. Tieu, K., and Viola, P. "Boosting image retrieval.” Proc. IEEE Conf. on Computer Vision and Pattern Recognition, vol. 1,pp. 228235,2000 .

[33]. X. S. Zhou and T. S. Huang. "Small sample learning duringmultimedia retrieval using biasmap."Proceedings of IEEEInternational Conference on Computer Vision and PatternRecognition (CVPR), Hawaii, Dec, 2001.

[34]. Hui Hui Wang, Dzulkifli Mohamad, N.A. Ismail

[35]. T. Dharani And I. Laurence Aroquiaraj. "A Survey on Content Based Image Retrieval" Proceedings of the 2013 International Conference on Pattern Recognition, Informatics and Mobile Engineering, February 21 -22

[36]. Hui Hui Wang, Dzulkifli Mohamad, N.A. Ismail. "Approaches, Challenges and Future Direction of Image Retrieval" JOURNAL OF COMPUTING, VOLUME 2, ISSUE 6, JUNE 2010, ISSN 2151-9617 